man nicht in der Lage war, sich einen zureichenden Begrifi von der Intensität der Radiumstrahlen zu bilden, und somit von falschen Vorstellungen beherrscht wurde. Den Befürchtungen von zu intensiver und schädlicher Wirkung wird heute dadurch vorgebeugt, daß nian gelernt hat, die Radiumstrahlen zu dosieren. Wo eine zu intensive Einwirkung möglicherweise von üblen Folgen begleitet sein könnte, ist es wertvoll, die radioaktive Kraft des anzuwendenden Präparates vorher zu bestimnen. Versuche und Erfahrung haben gelehrt, dal intensive und. langdauernde Finwirkung von Strahlen auf das lebende Geweb? Ulzerationen und. Entrïndungen hervorrufen kann, und deswegen ist es nützlich zu wissen, bis zu welchem Intensitätsgrade wir mit d.em Radium uns vortrauen dürfen. London (Petersburg) hat, um zu beweisen, wie gering die Radium ausstrahlende Fläche sein darf, um einen. Effekt zu crzielen, auf den Unterarn von verschiedenen Personen $18 \mathrm{~nm} g$ in einer Schachtel verschlossenes Radium appliziert, und zwar auf die Dauer von $1 / 8-5$ Minuten, und dabei stellte es sich heraus, daß bereits $1 / 4$ Minute genügte, um einen dauernden Hautfleck von rotbrauner Farbe zu erzielen. Ader nur dort, wo wir vor zu intensiver Einwirkung die Patienten schützen wollen, ist die ungefähre Kenntnis der anzuwendenden Intensität ein notwendiges Erfordernis der Vorsicht.

Wir dürfen aber auch den Wert und die Bedeutung der Dosierung nicht ïberschätzen, denn $\mathrm{zu}$ einer notwendigen Vorausbestinmung der erforderlichen Radioaktivität gehört auch die Berücksichtigung der genauen Wirkungsweise des Radiuns, der Flächenausdehnung der Strahlenquelle, der Dauer der Anwendung und der Flächenberechnung des zu bestrahlenden Gebietes. Die Kenntnis aller dieser Moniente und die Beriicksichtigung jedes einzelnen würde erst die Möglichkeit geben, die anzuwendende Dosis vorherzubestimmen. Inı übrigen herrscht beim Bestinımen der Radioaktivität selbst in den Kreisen der erfahrenen Radiotherapeuten eine ziemlich große Verwirrung.

Man hat das Uran als Maßeinheit erklärt, läßt aber mcist

\section{Die Behandlung inoperabler Karzinome des Uterus mit Radiumstrahlen.')}

\section{Von San.-Rat Dr. E. Arendt in Gharlottenburg.}

Dem Worte Radiun haftet noch immer etwas Mystisches an, und wenn auch durch unablässiges Arbeiten zahlreicher Physiker und Biologen die geheimnisvolle Kraft dieses Edelnetalls in nannigfacher Richtung geklärt worden ist, so befindet sich die therapeutische Verwertung dieses neuen Mittels fast noch in Anfangsstadium. Durch das seltene Vorkommen und den dadurch bedingten hohen Preis dieser Substanz. ist die Zahl der Forscher begrenzt, sodaß es nur Wenigen vergönnt ist, therapeutische Versuche mit dem Radium anzustellen. Die mit großem Kapital arbeitende Industrie hat hier nun helfend eingegriffen. Was sie uns Aerzten aber bietet, ist nur ein Teil der geheimnisvollen Kraft, und zwar nur die Emanation. Dieses Gas, das dem Radium entströmt, kann aufgefangen, an die verschiedensten Elemente gebunden und auch an anderem Ort und zu anderer Zeit als an der Stätte und im Augenblick des Entstehens verwertet werden. Diese wertvolle Eigenschaft der Emanation hat eine ungeheure Anzahl von Arbeiten gezeitigt, die zum Teil wertvoll, zum Teil aber in bezug auf Erfolg und Wirkungsweise von Phantasie und Optimismus beeinflußt sind. Die Emanationstherapie, wie sie in der ärztlichen Verwertung bei der Behandlung der Krankheiten benannt wird, kann auch für gynäkologische Zwecke Verwendung finden; ich komme darauf noch später zurück.

Von besonderem Interesse für die Gynäkologie ist und bleibt aber die Verwertung der Radiumstrahlen und findet in glänzender, ungeahnter Weise dort Verwendung, wo unsere bisherigen Methoden, Medikamente und das chirurgische Messer nicht mehr ausreichen. Hatte man sich früher auch gescheut, die reinen Radiumsalze, abgesehen von ihrer Kostspieligkeit, anzuwenden, so hatte dies besonders darin seinen Grund, daß

1) Vortrag, gehalten auf dem 14. Kongreß der Deutschen Gesellschaft für Gynäkologie in München vom 7.-10. Junj 1911. (Diskussion hierzu in No. 28, S. 1335.) statt der gemeinten Flächeneinheit die Gewichtseinheit gelten, wodurch in vielen Fällen eine große Differenz in der Bezeichnung entsteht.

Ich habe den Eindruck, daß offenbar die Angabe der Dosierung der Therapie nur den Schein der Wissenschaftlichkeit verleiht. Insbesondere möchte ich dieses bei der Anwendung der durch Emanation radioaktivierten Präparate behaupten. Diese verlieren meist in ganz kurzer Zeit die induzierte Radioaktivität; die anfänglich genaue Dosierung hat alsdann ihren Zweck verfehlt und führt $\mathrm{zu}$ irrtïmlichen Anschauungen.

Dort, wo unter Benutzung von Radiunssalzen radiunhaltige Apparate angewandt werden, will ich die Berechtigung einer Aktivitätsbestimmung nicht in Abrede stellen. Doch dürfen wir dieser nicht allzu große Bedev.tung beimessen und nicht etwa erklären:. keine Radiumtherapie ohne Dosierung.

Ich habe nun weder die reinen Radiumsalze, noch auch die mit unlöslichen Salzen hergestellten Apparate benutzt, sondern bediente mich jenes Urmittels, aus dem das Ehepaar Curie das Radium gewonnen hat, und zwar der gepochten Joachinsthaler Uranpechblende, die durch ein besonderes Reinigungs- und vorbereitendes Konzentrierungsverfahren sich für meine Anwendungsweise vorzüglich eignet.

Was sie besonders wertroll macht, das ist, daß mein Präparat auch noch jene Strahlen enthält, die bei der Darstellung des reinen Radiums verloren gegangen sind. Das bedarf wohl einiger Worte der Aufklärung. Das Ehepaar Curie konnte durch ein umständliches chemisch-analytisches Verfahren von dem Urandioxyd auch das mit ihm verbundene Wismut trennen und feststellen, daß dieses 400 mal so stark radiooktiv war als das Cran selbst. Da das Wismut sonst nicht radioaktiv ist, so lag die Vermutung nahe, daß in dem in der Uranpechblende vorhandenen Wismut noch ein stark aktiver Körper enthalten sein mïsse; diesen hypothetischen Stoff nannten sie Poloniun. Aber außer diesem radioaktiven Stoff sind noch andere Bestandteile, die Radioaktivität enthalten, in der Uranpechblende nachzuweisen, z. B. das Actinium. So wenig wir auch in der Lage sind, die Behauptungen der Physiker in diesen Angaben nachzuprüfen, und so sehr wir uns besonders hierin auf ihre Autom 
rität verlassen müssen, so zeigen doch die Erfahrungen und Versuche, daß die Kombination verschiedener radioaktiver Elemente, die in der Uranpechblende außer dem Radium noch enthalten sind, eine besondere wirkungsvolle Kraft in sich schließen. Ich habe nun vor der Anwendung der Uranpechblende, die mir im reinsten Zustande zu. Gebote steht, die Prüfung auf Radioalktivität durch die Einwirkung auf die photographische Platte vorgenommen. In vielen Fällen fügte ich auch noch der Sicherheit wegen die elektrometrische Methode zum Nachweis hinzu, verzichtete aber auf die jedesmalige Dosierung der anzuwendenden Gabe. Dies ist begreiflich, weil ich bei den einzelnen Patientinnen verschieden große Quantitäten anwandte, und somit, dem Umfang entsprechend verschieden große Strahlenquellen, und weil ferner die zu bestrahlenden Flächen außerordentlich differierten. Aber damit wäre auch garnichts gewonnen gewesen, da mein Verfahren die Befürchtung einer $\mathrm{zu}$ intensiven Einwirkung nicht aufkommen ließ und die Dosierung nicht erforderlich machte. Während bei der Anwendung der reinen Radiumsalze auf das peinlichste die Dauer der Bestrahlung abgemessen werden muß, konnte ich die Uranpechblende vnbegrenzt lange zur Anwendung bringen. Tch konnte - und darin liegt ein besonderer Vorzug -_- den Mangel der Intensität der Einwirkung durch die Daver der Anwendung kompensieren.

Die Uranpechblende, wenn auch nicht in der reinen, mir zu Gebote stehenden Form, ist bereits vor mir von anderen. angewandt worden.

Strobel gelang es, mittels gepulverter Pechblende große Psoriasisherde zur Abblassung zu bringen. Auch Neusser (Wien) machte Gebrauch von durch Uranpechblende radioaktiviertem Wasser; er verwandte dieses zu Umschlägen. Ferner hat Dautwitz Badewasser durch eingelegte Uranpechblenderïckstände aktiviert -- ein in der Gegend von Joachimsthal seit langer Zeit geübtes Heilverfahren --. vnd beobachtete Heilung oder Besserung bei chronischen Gelenkaffektionen und Neuralgien.

Bei Neusser und Dautwitz wirkte aber die Radiumemanation, die von der Uranpechblende ausging, bei Strobel die direkte Radiumbestrahlung.

Die Frage nach der Bezugsquelle meines Präparates liegt, sehr nahe und ist nach meiner ersten Publikation in der Berliner klinischen Wochenschrift oftmals an mich gerichtet worden. Leider bin ich nicht in der Lage, eine befriedigende Antwort zu geben, da das Erzbergwerk Joachimsthal aus mir nicht bekannten Gründen die Abgabe selbst der wenig gereinigten Uranpechblende verweigert.

Einen nicht vollwertigen Ersatz bin ich in der Lage statt dessen anzugeben. Fs ist möglich, radiumhaltige Kohle (feinst pulverisierte Pflanzen- oder Tierkohle) herzustellen, die eine bestimmte Quantität unlöslicher Radiumsalze enthält. Ist nun die Verteilung des unlöslichen Radiumsalzes mit der vorher quantitativ bestimmten Kohlenmasse auf das genaueste vorgenommen, so kann die Radioaktivität dieses Gemisches nach Uraneinheiten festgestellt werden. Dies ist ein Präparat, das dem meinigen ähnlich, aber nicht, das gebe ich zu, gleichwertig ist und das in derselben Weise mittels der Kondomfingerlinge in einer Umhüllung in. den Uteruskrater auf Karzinome gebracht werden kann. Es enthält selbstverständlich nicht die wertvollen Strahlen (Polonium, Actinium), die außer dem Radium noch in der Joachimsthaler Uranpechblende enthalten sind und deren Wirksamkeit ich sehr hoch einschätze.

Was nun meine Behandlung der Karzinome des Uterus anbetrifft, so bin ich von dem Grundsatze ausgegangen, daß die Karzinombehandlung in erster Linie eine operative sein muß. Bietet sie auch keine absolute Sicherheit vor Rezidiven, so sind wir doch gewiß, daß sie auf die radikalste Weise die Karzinomherde entfernt und somit für den Körper unschädlich macht. Nur die traurigsten, am weitesten vorgeschrittenen und für die operative Entfernung prognostisch von vornherein ungünstigsten Fälle konnten der Gegenstand meiner Behandlung mit den Bequerellstrahlen sein. Aber auch hier galt für mich die Regel: Kombination operativen Vorgehens und physikalischer Behandlung. Kurz, bei genauer Abwägung der Chancen der Operabilität durch radikale Entfernung des Organs und bei ev. Erkenntnis der Zwecklosigkeit dieses Vorgehens entschließe ich mich zu einer gründlichen Exkochleation aller karzinomatösen Massen; alsdann erfolgt sofort Ausbrennen mit glühendem Weißlicht und Einlegen von Gazestreifen mit $30 \%$ iger Chlorzinklösung oder $\mathbf{4 0} \%$ iger Zinkopyrinlösung, um bald darauf, d. h. nach zwei bis drei Tagen., die Anwendung der Uranpechblende vorzunehmen.

Auch Seelig (Franzensbad) hat bereits die Behandlung inoperabler Trmoren mit Radium versucht. - Er hat inoperable Karzinome mit Radium bestrahlt, indem er dieses etwa 24 Stınden liegen ließ. Seiner Angabe nach war kein besonderer Rückgang der Geschwulstmassen zu beobachten, aber eine bedentende Verminderung der Schmerzen. Es konnte selbstverständlich kein Erfolg bei dieser Anwendung erzielt werden, denn die Radiumstrahlen werden bereits von den ersten Schichten des Karzinomgewebes zum großen Teil absorbiert.

Chéron und Faber berichteten in der Société d'obstétrique de Paris im Jahre 1909 über Anwendung von radioaktivenı Schlamm in der Gynäkologie; sie haben alle möglichen gynäkologischen Erkrankungen mit diesem Mittel behandelt, in Form von Spïlıngen, Um schlägen und Bädern. Ihre Erfolge beruhten angeblich darauf, daß sie eine analgesierende, hämostatische und dekongestionierende Wirkung erzielten.

Petit warnt in der "Gynécologie", 1909, No. 4, vor allzu großen Hoffnungen in der Behandlung von Neubildungen mit Radium; Erfolg verspricht er sich nur bei Fibromen und Karzinomen.

Chéron und Rubens Duval (Société d'obstétrique de Paris 1909, berichten ïber eigene Beobachtıngen bei der Behandlung von Karzinomen des Uterus und der Vagina mittels Radiumstrahlen. Sie haben drei Fälle von Uteruskarzinomen und zwei Fälle von sekundärem Scheidenkarzinom behandelt. Sie erzielten in allen Fällen Schwinden von Schmerzen, Blutungen und Ausflu $B$ und eine fast vollständige Resorption der Geschwïlste, letztere in der Weise, daß statt des ,brüchigen, leicht blutenden Gewebes der Neubildung " nur "derbe Massen zu fühlen“" waren.

Mangiagalli (Mailand) berichtet auf dem V. Internationalen Kongreß für Geburtshilfe und Gynäkolngie in Petersburg, im September 1910, über die Therapie des nicht operablen Gebärmutterkrebses und erwähnt hierbei auch die Anwendung des Radiums in Verbindung mit anderen therapentischen Maßnahmen. $\mathrm{Er}$ will in einigen Fällen gewisse Erfolge gesehen haben, indem der Uterus nach einer solchen Behandlung wieder beweglich wurde und Krebse, die anfangs für inoperabel galten, von ihm später operiert werden konnten.

Fräulein Dr. Lurrié (Petersburg) hat auf demselben Kongreß über die Einwirkung von Radium auf Mäusekarzinome berichtet. Ihrer Angabe nach wurde das Radium direkt auf die Tunoren gelegt (im Behälter), die Dauer der Séancen war 5-60 Minuten ind deren Zahl 4-14. Sie kommt zu der Erkenntnis, daß das Radium in kleinen Dosen eine irritative Einwirkung auf die Krebszellen habe, in größeren sie zerstören könne. Der Wert dieser Beobachtungen leidet durch die Art der Anwendung des Radiums, das auf den Tunor selbst appliziert worden ist, soda $\beta$ also die Strahlenwirkung nicht voll und ganz bis zur Basis des Tumors, $d . h$. bis in das gesunde Gewebe gelangen konnte.

Einer besonderen Beachtung bedarf die Mitteilung, die Wickha m in seiner wertvollen Publikation in Gemeinschaft mit Degré über Be. handhung der Uteruskarzinome gemacht hat. So hoch auch die Arbeiten dieser beiden Forscher und ihre Verdienste um die wissenschaftliche Radiumbehandlung zu schätzen sind, so darf doch berechtigte und eingehende Kritik ihrer therapeutischen Resultate nicht schweigen. Die hohe Autorität beider Gelehrten fordert zur Begrïndung der geäußerten Zweifel an den publizierten Heilerfolgen heraus. Wickham

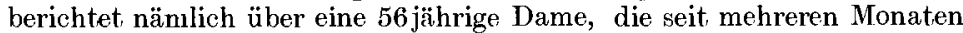
an einem Epitheliom des Uterus leidet. Sie hat Abgänge einer dïnnfliissig̀en, jauchigen, oft rosa gefärbten Masse, an der Zervikalportion sind schon Wucherungen mit scharfem Löffel entfernt worden, und der Uterus ist sehr groß, unbeweglich und schmerzhaft. Es wird die Patientin von Dr. Tuffier für inoperabel gehalten. Nach fünfmaliger Behandlung von 13stündiger Dauer, wobei der radium. haltige Apparat direkt auf die Wucherungen des Uterushalses gelegt wird, sind alle Wucherungen an der Portio verschwunden, und Dr. Tuffier ist in der Lage zu konstatieren, daB der Uterus viel weicher geworden und nicht mehr im kleinen Becken fixiert sei. Einige weitere Sitzungen beseitigen das Karzinom vollständig, sodaß Patientin fünf Monate, nachdem das Karzinom zuerst entdeckt worden ist, operiert werden konnte. Hier also soll die Radiumbehandlung die Rückbildung des Tumors bewirkt haben. Es nimmt dies um so mehr wunder, weil meiner Ansicht nach das Verfahren kein rationelles war. Wollen wir anf Karzinome einwirken, so können wir es nicht dadurch, daß wir die äußeren Schichten in Angriff nehmen und bis zu deren Zerstörung den tieferen Schichten, die an das Gesunde grenzen, die Möglichkeit geben, weiter 
in destruierender Weise, wie es die Malignität mit sich bringt, vorzudringen.

Noch märchenhafter klingt der Bericht über eine zweite Patientin von 48 Jahren. Hier bestand eine Neubildung, die durch histologische Untersuchungen als karzinomatös festgestellt wurde; 3/4 Jahre nach Feststellung der Malignität der Neubildung wurde von Dr. Tuffier operiert. Das Rezidiv trat 1/2 Jahr später auf, und nun begann die Radiumbehandlung. In neun Sitzungen wurde das Rezidiv beseitigt. Die Notwendigkeit der Dosierung, auf die der Autor sonst ganz besonderes Gewicht legt. scheint auch für diesen Fall außer acht geblieben zu sein, denn sonst hätte der ausführliche und gewissenhafte Bericht auch diese gewiß erwähnt.

Diese traumhaften Erfolge verblüffen jeden Erfahrenen. Und

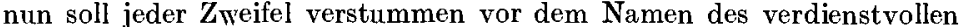
französischen Forschers, Wickha m, und der Autorität des Opsrateurs?

Mein Instrumentarium ist einfach. Ich benvtze die gepochte, durch ein besonderes Verfahren gereinigte Uranpechblende und richte mich in der Anwendung der Quantität ganz nach der Größe und dem Umfang des von mir durch Exkochleation gebildeten Kraters. Verschieden sind daher die Quantität der Substanz und folglich auch die angewendeten Uraneinheiten. Was meiner Anwendungsweise den Vorzug gibt vor der der Herren Wickham und Degré, ist, daß ich soviel als möglich auf die Stelle wirke, an der die Karzinommassen vor der Exkochleation das Gesunde berührt haben, an der also die Malignität normalerweise vorzuschreiten beginnt. Nach Wickham und Degré haben die Strahlen eine destruierende und modifizierende Wirkung. Die erstere ist ja keineswegs zu. leugnen und offenbart sich dem Auge; mit ihr in Verbindung steht die Entzündung. Es ist dies leicht verständlich, wenn man sich folgendes vergegenwärtigt. Eine kleine Flamme, auf einen geringfügigen Gewebsbezirk gerichtet, wird eine relativ kleine Ulzeration und. einen entsprechenden Entzündungshof hervorrufen. Die gesamte Wärmeenergie wirkt konzentriert. Verteilt man nun dieselbe Wärmeenergie auf eine große Fläche, so wird nur die Temperatur dieser Fläche erhöht, und man beobachtet den für das Gewebe spezifischen Wärmereiz, der statt destruierend sehr wohltätig wirken kann.

Auch das Radium entwickelt Wärme, aber bei kleiner intensiver Strahlenquelle wirkt sie destruierend und entzündend; geht aber die Strahlung von großer, umfangreicher Fläche aus, so wirkt sie als heilbringender Reiz. Wie weit die $\alpha$-Strahlen $\ddot{u}$ berhaupt destruierend auf die Karzinommassen wirken, wissen wir nicht. Sicher ist, daß die a-Strahlen kaum zur Wirkung kommen und schon in den obersten Schichten des Tumors absorbiert werden; von $\beta$-Strahlen dringen nur die harten ein wenig tiefer hinein, während die $\gamma$-Strahlen weit hinaus über die Karzinommassen selbst in die Tiefe gehen. Aber das eine bleibt dabei zu berücksichtigen: von allen Strahlen, die von dem Radium ausgehen, betragen die $\gamma$-Strahlen nur $1 \%$. Was nun der Ausdruck ,,modifizieren" im Sinne von Wickham und Degré bedeuten soll, ist mir nicht klar. Ich bestreite aber, daß Karzinomzellen, und wären es auch die jüngst entstandenen, die nahe dem Gesunden gelegen sind, zu normalen Zellen modifiziert werden können. So wertvoll das Agens auch ist, so kann man doch immerhin nur die Heilung durch eine irritative Wirkung und durch Bildung von Granulationsmassen erzielen. Dieses erstrebe ich mit meiner Behandlung. Ich weiß, daß die Masse der Strahlen nur gering sein kann, die von der Uranpechblende ausgehen, aber direkt auf die Basis des exkochleierten Tumors wirken muß. Aber nicht allein der Reiz der Strahlen ist es, der eine heilende Wirkung ausübt, es muß sich an den Wänden des Kraters auch Emanation niederschlagen und mit dem Gewebe innig verbinden. Wissen wir doch von der Emanation, daß sie in jedes Gewebe eindringt und. umso fester und. länger haftet, wenn ihr die Möglichkeit des Verflüchtigens durch Abschluß von der Außenwelt genommen ist. Tagelang halten sich die Emanationen an derWand des Kraters, und immer von neuem macht sich eine Emanationswirkung geltend. Nur so können wir uns das Rätsel der Emanationswirkung erklären. Mit dieser Erklärung ist es begreiflich und verständlich, daß manche Forscher von einer Nachwirkung sprechen, die im Augenblick nicht sichtbar und erkennbar ist. Ich habe diese akkumulative Wirkung auch bei anderen gynäkologischen Erkrankungen, über die ich an anderer Stelle berichten werde, beobachten können. Ich halte die Apparate, die Wickham und Degré anwenden, sicherlich für sehr wirkungsvoll; sie sind die Erzeugnisse unablässiger wissenschaftlicher Arbeit und werden ihre Anwendung finden auf vielen anderen Gebieten, aber für gynäkclogische Zwecke erscheinen sie mir unbrauchbar, vielleicht auch gefährlich.

Wir wissen aus unseren früheren Erfahrungen, daß der mechanische Reiz von Stäbchen, eingeführt in die Uterushöhle, Schmerzen hervorruft und. Reizzustände schafft, die den ganzen weiblichen Organismus angreifen. Die Beseitigung von Uterusstäbchen aus der gynäkologischen Therapie bedeutet ein Fortschreiten, die Wiedereinführung kann keinen Nutzen bringen.

Ist der Uterus karzinomatös erkrankt, so ist die schnellste operative Entfernung desselben oder gründliche Exkochleation ein absolutes Erfordernis; ein längeres Zögern und. Hinziehen mit Anwendungen von Reiz schaffenden Instrumenten kann nur zum Nachteil gereichen.

Ein besonderes Augenmerk lege ich auf die Anwendung der Asepsis. Die jedesmalige Behandlung erfordert eine Desinfektion des karzinomatösen Kraters. Ich nehme sie mit Wasserstoffsuperoxyd vor und bringe alsdann die Uranpechblende in Kondomfingerlingen, die ich in der verschiedensten Länge und Weite besitze, entsprechend der Weite der Höhle, in diese hinein. Der Kondomfingerling soll steril sein; er wird außerdem in sterile Jod.cformgaze eingewickelt. Die Umhüllung mit einer einfachen Lage von Jodoformgaze, die ein wenig in die Vagina hineinragt, sichert gleichzeitig die leichtere Entfernung des Präparates beim Auswechseln.

Ich bin der festen Ueberzeugung, einer großen AnzahI Frauen ist durch die Radiumbehandlung das Leben verlängert worden, und auch diejenigen, die dem Karzinom zum Opfer gefallen sind, haben sicherlich die letzten Tage ihres Lebens nicht das Empfinden des Dahinsiechens gehabt, wie avch die Umgebung selbst bis zum nahen Ende die Hoffnung auf die Heilwirkung des Radiums bewahrt. Dies erscheint als ein außerordentlicher Fortschritt, wenn wir bedenken, unter weIchen Zuständen die Karzinomkranken sonst zu. leiden haben und wie oft die Umgebung die Stunde der Erlösung von den Schmerzen für die Patientin herbeisehnt.

Was die Erfolge anbetrifft, so können die relativ wenigen Fälle eines cinzelnen Beobachters statistisch nicht verwertet werden; auch bleibt dabei zu erwägen, daß es sich ja um die traurigsten, am weitesten vorgeschrittenen Fälle handelt.

Von den 26 karzinomatösen sind 18 inoperable Fälle gewesen, 10 sind gestorben, 8 leben und sind noch in meiner Behandlung; von diesen sind 6 in gutem Allgemeinzustande, 2 werden voraussichtlich bald zugrunde gehen, 3 sind. trotz ihres Karzinoms arbeitsfähig, auch die andern 3 machen den Eindruck gesunder Individuen.

In 8 Fällen ist Totalexstirpation (5 abdominal und 3 vaginal) vorangegangen. 4 kamen mit Rezidiv in Behandlung, darunter 3 nach abdominaler Totalexstirpation, aber in desolatem Zustande; sie sind in kurzer Zeit gestorben. In 4 Fällen habe ich im Anschluß an die Totalexstirpation die Radiumbehandlung begonnen; darunter hat nur eine Patientin ein Rezidiv, während die anderen vorläufig noch rezidivfrei sind. Ich habe den Eindruck, daß die Radiumbehandlung post operationem einen gewissen Schutz vor Rezidiven gewährt. Ich beginne damit gleich am dritten Tage, an welchem ich die Operierte zum ersten Male aufstehen lasse.

Unter den Lebenden sind mehrere, die über zwei Jahre bereits in meiner Behandlung sind, und als besonderen Erfolg stelle ich fest, daß einzelne, die in desolatem Zustande in die Behandlung kamen, zwar von ihrem Karzinom nicht radikal befreit, soweit wir es eben feststellen können, aber frei von Beschwerden und Schmerzen sind. Wenn auch nicht Heilung erzielt ist, so befinden sich doch die Behandelten in einem Zustande, der an Heilung grenzt. Als hervorragender Effekt der ganzen Radiumbehandlung - fast ohne Ausnahme ist zu konstatieren, daß in kurzer Zeit die Beseitigung der Kardinalsymptome ,Odor, Fluor, Dolor" beobachtet werden kann. Selbstverständlich, und das entspricht den Erfahrungen aller Gynäkologen, sind die ungünstigsten Fälle diejenigen Karzinomfälle, die Frauen in jüngeren Jahren (zwischen 20 
bis 40) betreffen. Anfängliche Besserung und doch Auftreten eines schmerzlosen Siechtums. In den vollsaftigen, meist noch menstruierenden Personen mit der noch vorhandenen monatlichen Fluxion zu den Unterleibsorganen tritt als schädlich einwirkcndes Moment immer wieder, selbst dort, wo lokale Herde sich zv reinigen beginnen und neve Granulationsmassen emporschießen, ein Fortschreiten der Malignität durch Metastasen in den entfernteren Organen auf.

Die Therapie erfordert außerordentliche Vorsicht; irgendein Akzidens bedarf der genauesten Untersu.chung. Jede Abweichung von den normalen Funktionen der einzelnen Organe kann indirekt durch die Einwirkung der Bequerellstrahlen hervorgerufen werden. Fieber, Schmerzen erfordern zeitweilige Entfernung der Substanz. Das Haupterfordernis aber der Behandlung ist absolute Sauberkeit.

Zum Schlusse bomerke ich, daß die Radiotherapie bei den Uteruskarzinomen, soweit wir bis jetzt in der Lage sind, die Wirkungsweise zu verstehen, uns keine begründete Hoffnung auf wirkliche Heilerfolge gibt, wenn sie auch in manchen Fällen Zustände schafft, die an Heilung grenzen. Ich wiederhole daher ausdrücklich, um jedem Mißverständnis und jeder falschen Devtung meines Vorgehens vorzubeugen, ich vertrete aus voller Ueberzeugung die Ansicht, daß in erster Linie die Radikaloperation anzustreben ist, und halte es für ein jeder Wissenschaftlichkeit widerstreitendes Verfahren, für einen verwerflichen Optimismus, die Behandlung jedes Uteruskarzinomes unterschiedslos mit der Radiotherapie zu beginnen.

Es ist zu hoffen, daß die fortschreitende Technik in der Radiotherapie unsere Behandlungsresultate verbessern wird. Noch stehen wir am Anfange der Anwendung dieser neuen physikalischen Heilmethode auf den verschiedensten Spezialgebieten des ärztlichen Schaffens, noch sind wir auf dem Wege des Versuchens und Experimentierens, noch ist das Indikationsgebiet kein umgrenztes. Soll daher die Radiotherapie in den Händen wissenschaftlicher Aerzte bleiben, so müssen die Berichte über Heilresultate frei bleiben von jeder Uebertreibung und einem gesunden wissenschaftlichen Skeptizismus standhalten. Nur so werden wir diese neue, wertvolle Behandlungsmethode vor Diskreditierung schützen und ernste Mitarbeiter auf diesem noch wenig bebauten Felde der Arbeit gewinnen.

Zusammenfassung. 1. Die genaue Dosierung ist nur dann ein Erfordernis, wenn wir hohe Intensitäten anwenden.

2. Hohe Intensitäten einer kleinen Strahlenquelle sind zu meiden und durch umfangreiche Flächen von geringer Radioaktivität $\mathrm{zu}$ ersetzen.

3. Die Dauerwirkung geringer Radioaktivität ist der kurz dauernden Einwirkung hoher Intensität vorzuziehen.

4. Die Energie des Radiums, Strahlen und Emanation, ist für das Karzinom des Uterus kein. Heilmittel par excellence, aber besser und wirkungsvoller als jedes uns sonst bekannte Medikament oder irgendeine der üblichen physikalischen Heilmethoden.

5. Die Radiotherapie findet ihre Indikation:

a) bei aussichtsloser Radikaloperation, und zwar dann nach vorangegangener Entfernung alles Karzinomatösen.

b) als Schuttz vor Rezidiven nach der erweiterten Radikaloperation. 